\title{
Comment on the relationship between an exact two-photon solution and a two-photon vector model
}

\author{
D. Grischkowsky* \\ IBM Thomas J. Watson Research Center, Yorktown Heights, New York 10598 \\ Richard G. Brewer ${ }^{\dagger}$ \\ IBM Research Laboratory, San Jose, California 95193 \\ (Received 19 October 1976)
}

\begin{abstract}
The exact pulse solution of Brewer and Hahn for optical two-photon transitions is shown to reduce in the adiabatic limit to the two-photon vector model used by Grischkowsky, Loy, and Liao. The connection between the two approaches is explained and the complementary relationship between the two papers is discussed.
\end{abstract}

\section{INTRODUCTION}

The field of coherent two-photon spectroscopy has experienced vigorous growth in the last few years, and several theoretical studies of the optical two-photon resonance problem have appeared. Because of the computational complexity, even informed readers might find the relation between various papers obscure. Furthermore, there is a profusion of notation.

This Comment addresses this problem by comparing the exact pulse solution of Brewer and Hahn $(\mathrm{BH})^{1}$ for the two-photon resonance of the three-level atom with the adiabatic two-photon vector model used by Grischkowsky, Loy, and Liao (GLL). ${ }^{2}$ It will be noticed that the BH paper stresses the case where light pulses, of frequency $\Omega_{1}$ and $\Omega_{2}$, are applied suddenly (nonadiabatically) with respect to the frequency offset $\Delta$ of the intermediate atomic state (see Fig. 1). On the other hand, the GLL approach considers the light to be applied slowly or adiabatically with respect to $\Delta$. This difference accounts for the presence of high-frequency ringing terms which appear in the BH solution that are not present in the GLL result. We will show that the $\mathrm{BH}$ result can also yield adiabatic solutions (without ringing) as $\Delta$ is arbitrarily increased, and therefore can be compared directly with the GLL calculation. In fact, both adiabatic solutions in the appropriate limit are found to be identical. In order to keep this Comment to a reasonable length, the treatment relies heavily on the details given in the $\mathrm{BH}$ and GLL papers. Assuming that the reader has these papers at hand, we do not define all of the notation and quantities introduced.

\section{THE BREWER AND HAHN SOLUTION}

In this section we first review the exact pulse solution of $\mathrm{BH}$. Then, we extend it to cover the case where the two-photon resonant pulses are applied adiabatically with respect to the intermediate state. In the notation of $\mathrm{BH}$, the system under consideration is the three-level atom of Fig. 1. The atom is irradiated by counter-propagating light pulses with field strengths $E_{1}$ and $E_{2}$ and frequencies $\Omega_{1}$ and $\Omega_{2}$. The BH paper shows that the set of coupled equations describing this physical situation is equivalent to the reduced set of Bloch equations:

$$
\begin{aligned}
& \dot{U}-\Delta V=0, \\
& \dot{V}+\Delta U+\epsilon W=0, \\
& \dot{W}-\epsilon V=0,
\end{aligned}
$$

when the two-photon resonance condition

$$
\Omega_{1}+\Omega_{2}=\omega_{21}=\omega_{2}-\omega_{1}
$$

is satisfied and when the applied fields are constant, i.e.,

$$
\dot{\alpha}=\dot{\beta}=\dot{\epsilon}=0 .
$$

The quantities $U, V$, and $W$ are linear combina-

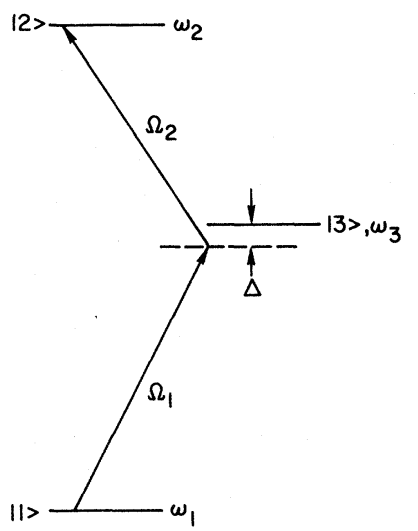

FIG. 1. Energy-level diagram for the two-photon transition of the three-level atom in the BH notation. (Note that levels 1 and 2 are interchanged in Fig. 3.) 
tions of the density matrix elements $\rho_{i j}(i, j=1,2,3)$ associated with the three atomic states of Fig. 1. Equation (1) can also be written as

$$
\frac{d \overrightarrow{\mathrm{B}}}{d t}=\overrightarrow{\mathrm{B}} \times \vec{\gamma}
$$

which represents the precessional motion (Fig. 2) of a two-photon Bloch vector $B(U, V, W)$ about an effective field

$$
\gamma=\left(\Delta^{2}+\epsilon^{2}\right)^{1 / 2},
$$

where the two-photon Rabi frequency

$$
\epsilon=2\left(\alpha^{2}+\beta^{2}\right)^{1 / 2}
$$

is a function of the one-photon Rabi terms

$$
\alpha=\mu_{13} E_{1} / 2 \hbar, \quad \beta=\mu_{23} E_{2} / 2 \hbar .
$$

For the purpose of this discussion, the detuning parameter $\Delta$ is defined

$$
\Delta=\Omega_{1}-\omega_{31}=\omega_{23}-\Omega_{2},
$$

appropriate to atoms with zero Doppler shift, $k v_{z}=0$, where we assume $\Delta$ to be negative, consistent with Fig. 1.

For the case where the initial conditions at $t=0$ are appropriate for the two-photon resonant light hating been applied nonadiabatically with respect to the intermediate state, Eq. (1) has the solution

$$
\begin{aligned}
& U(t)=-W(0) \sin \phi \cos \phi[\cos (\gamma t)-1], \\
& V(t)=-W(0) \sin \phi \sin (\gamma t),
\end{aligned}
$$

$$
W(t)=W(0)\left\{1+\sin ^{2} \phi[\cos (\gamma t)-1]\right\},
$$

with

$$
\begin{aligned}
& \sin \phi=\epsilon / \gamma, \\
& \cos \phi=-\Delta / \gamma .
\end{aligned}
$$

In Eqs. (3), we have introduced the angle $\phi$ between the axis of the "rotating frame" and the precession axis of the two-photon Bloch vector $\overrightarrow{\mathrm{B}}$ $=(U, V, W)$. The precessional motion of $\overrightarrow{\mathrm{B}}$ is shown in Fig. 2(a), where $U$ is the projection of $\vec{B}$ on the $1^{\prime}$ axis, $V$ is the projection on the $2^{\prime}$ axis, and $W$ is the projection on the $3^{\prime}$ axis. Figure 2(a) describes the situation where initially $\vec{B}$ is pointing along the $+3^{\prime}$ axis. Then, at $t=0$ the two-photon resonant pulses are suddenly applied and $\vec{B}$ precesses about the precession axis $\vec{\gamma} / \gamma$ with angular frequency $\gamma$. This motion leads to the nonadiabatic solutions of $\mathrm{BH}$ for the following quantities:

$$
\begin{aligned}
u_{12}(t)= & \frac{8 \alpha \beta K}{\epsilon}(\cos \gamma t-1)-\frac{\alpha \beta}{\delta}\left(\frac{\alpha^{2}-\beta^{2}}{\alpha^{2}+\beta^{2}}\right) w_{12}(0) \\
& \times\left(\frac{\cos (\delta-\Delta / 2) t-1}{\delta-\Delta / 2}+\frac{\cos (\delta+\Delta / 2) t-1}{\delta+\Delta / 2}\right),
\end{aligned}
$$

$$
v_{12}(t)=\frac{\alpha \beta w_{12}(0)}{\delta}\left(\frac{\sin (\delta-\Delta / 2) t}{\delta-\Delta / 2}-\frac{\sin (\delta+\Delta / 2) t}{\delta+\Delta / 2}\right)
$$

$$
w_{12}(t)=w_{12}(0)+\frac{2 \alpha^{2} \beta^{2}}{\delta\left(\alpha^{2}+\beta^{2}\right)} w_{12}(0)\left(\frac{\cos (\delta-\Delta / 2) t-1}{(\delta-\Delta / 2)}+\frac{\cos (\delta+\Delta / 2) t-1}{(\delta+\Delta / 2)}\right)+\frac{K \epsilon\left(\alpha^{2}-\beta^{2}\right)}{\left(\alpha^{2}+\beta^{2}\right)}(\cos \gamma t-1),
$$

with

$$
\delta=\left(\alpha^{2}+\beta^{2}+\Delta^{2} / 4\right)^{1 / 2} .
$$

In terms of the density matrix elements of the initial and final states, levels 1 and 2 of the twophoton process, these quantities are defined as

$$
\begin{aligned}
& u_{12}=\tilde{\rho}_{12}+\tilde{\rho}_{21}, \\
& i v_{12}=\tilde{\rho}_{12}-\tilde{\rho}_{21}, \\
& w_{12}=\rho_{11}-\rho_{22} .
\end{aligned}
$$

For negative $\Delta$, we see that high-frequency ringing terms appear at frequencies $\gamma$ and $\delta-\Delta / 2$ due to the sudden application of the driving fields.

Notice that the nonadiabatic case can be represented by a reduced three-level Bloch vector model through Eq. (2a), but not through Eqs. (4a)-(4c) which have no simple geometrical interpretation. We will now show that Eqs. (4a)-(4c) reduce in the

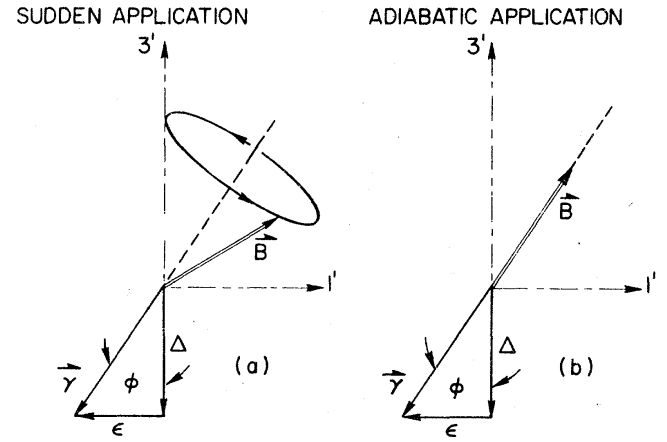

FIG. 2. BH vector model. (a) Precession of the Bloch vector $\overrightarrow{\mathrm{B}}$ when the two-photon resonant fields are applied suddenly with respect to the intermediate state. (b) Position of the Bloch vector $\vec{B}$ when the two-photon resonant fields are applied adiabatically with respect to the intermediate state. For the purpose of discussion, we assume $\Delta<0$. 
adiabatic limit to the two-photon vector model of GLL. In effect, this becomes a two-level Bloch vector, involving only levels 1 and 2 , and applies when the frequency offset $\Delta$ of the intermediate level is sufficiently large. For this case, the rise time of the two-photon resonant pulses must be long compared to the period of the frequency offset $\Delta$. For this purpose, we obtain the time-independent adiabatic solution of Eq. (1):

$$
\begin{aligned}
& U(t)=W(0) \sin \phi, \\
& V(t)=0, \\
& W(t)=W(0) \cos \phi,
\end{aligned}
$$

which is illustrated in Fig. 2(b). Here, the Bloch vector remains aligned along the precession axis. Using Eq. (5) above and Eqs. (9) and (14) of BH, we obtain

$$
\begin{aligned}
& u_{12}(t)=-\frac{\alpha \beta}{\delta}\left(\frac{\alpha^{2}-\beta^{2}}{\alpha^{2}+\beta^{2}}\right) w_{12}(0)\left(\frac{\cos (\delta+\Delta / 2) t-1}{\delta+\Delta / 2}\right), \\
& v_{12}(t)=\frac{-\alpha \beta}{\delta} w_{12}(0) \frac{\sin (\delta+\Delta / 2) t}{(\delta+\Delta / 2)}, \\
& w_{12}(t)=w_{12}(0)+\frac{2 \alpha^{2} \beta^{2}}{\delta\left(\alpha^{2}+\beta^{2}\right)} w_{12}(0)\left(\frac{\cos (\delta+\Delta / 2) t-1}{\delta+\Delta / 2}\right) .
\end{aligned}
$$

The absence of the high-frequency terms in Eq. (6) is now evident. Only slowly varying oscillations appar for this adiabatic case, and these are precisely the same terms contained in Eq. (4). Later, we will compare Eq. (6) with the corresponding quantities of the two-photon vector model of GLL.

\section{THE TWO-PHOTON VECTOR MODEL OF GRISCHKOWSKY, LOY, AND LIAO}

The vector model of GLL will now be reviewed. This model always assumes that the two-photon pulses are applied adiabatically with respect to the frequency offset of the intermediate state, but the model makes no assumption whether the pulses are applied adiabatically or nonadiabatically with respect to the two-photon resonance. The notation of GLL is illustrated in Fig. 3 and will be used exclusively in this section. As described in GLL, the precession of the two-photon $\overrightarrow{\mathbf{r}}$ vector about the $\vec{\gamma}$ vector is given by

$$
\frac{\partial \overrightarrow{\mathrm{r}}}{\partial t}=\vec{\gamma} \times \overrightarrow{\mathrm{r}}
$$

and is shown in Fig. 4. The components of $\overrightarrow{\mathbf{r}}$ are given in terms of the density matrix as

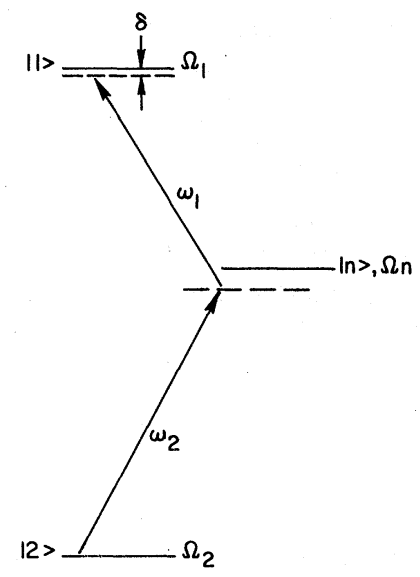

FIG. 3. Energy-level diagram for the two-photon transition of the three-level atom in the GLL notation.

$$
\begin{aligned}
& r_{1}=\rho_{12}^{\prime \prime}+\rho_{21}^{\prime \prime}, \\
& r_{2}=i\left(\rho_{12}^{\prime \prime}-\rho_{21}^{\prime \prime}\right), \\
& r_{3}=\rho_{11}^{\prime \prime}-\rho_{22}^{\prime \prime},
\end{aligned}
$$

and the components of $\vec{\gamma}$ are equal to

$$
\begin{aligned}
& \gamma_{1}=-\kappa \mathcal{E}_{1} \mathcal{E}_{2}, \\
& \gamma_{2}=0, \\
& \gamma_{3}=\delta+\left(\Delta E_{1}-\Delta E_{2}\right) / \hbar,
\end{aligned}
$$

with

$$
\begin{aligned}
& \delta=\Omega_{12}-\left(\omega_{1}+\omega_{2}\right), \\
& \kappa=\frac{1}{2 \hbar^{2}}\left|p_{1 n} p_{n 2}\left(\frac{1}{\Omega_{n 2}-\omega_{1}}+\frac{1}{\Omega_{n 2}-\omega_{2}}\right)\right|, \\
& \Delta E_{1}=\left|p_{1 n} \mathcal{E}_{1}\right|^{2} /\left[4 \hbar\left(\Omega_{1 n}-\omega_{1}\right)\right], \\
& \Delta E_{2}=\left|p_{2 n} \mathcal{E}_{2}\right|^{2} /\left[4 \hbar\left(\omega_{2}-\Omega_{n 2}\right)\right] .
\end{aligned}
$$

We adapt to the adiabatic $\mathrm{BH}$ calculation of the

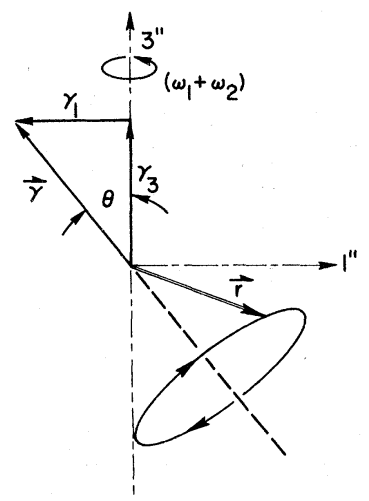

FIG. 4. GLL two-photon vector model. Precession of the $\overrightarrow{\mathbf{r}}$ vector about the $\vec{\gamma}$ vector when the two-photon resonant pulses are applied adiabatically with respect to the intermediate state, but suddenly with respect to the two-photon resonance. 
previous section, where at $t=0$ the two pulses are applied adiabatically with respect to the intermediate state, but nonadiabatically with respect to the two-photon resonance, i.e., $\delta=0$ corresponding to the two-photon resonance condition. To conform to the $\mathrm{BH}$ solution, we require that each radiation field ( $\omega_{1}$ for example) interacts with only one transition $\left(\Omega_{1 n}\right)$ and not the other $\left(\Omega_{n_{2}}\right)$. Furthermore, only one intermediate level is assumed, and it is labeled $n=3$. The solution for the motion of $\overrightarrow{\mathrm{r}}$ then becomes

$$
\begin{aligned}
& r_{1}=-\sin \theta \cos \theta(\cos \Gamma t-1), \\
& r_{2}=-\sin \theta \sin \Gamma t, \\
& r_{3}=-1-\sin ^{2} \theta(\cos \Gamma t-1),
\end{aligned}
$$

with

$$
\begin{aligned}
& \Gamma=\left(\gamma_{1}^{2}+\gamma_{2}^{2}+\gamma_{3}^{2}\right)^{1 / 2}, \\
& \sin \theta=-\gamma_{1} / \Gamma, \quad \cos \theta=\gamma_{3} / \Gamma .
\end{aligned}
$$

\section{RELATIONSHIP BETWEEN THE RESULTS BH AND GLL}

The connection between the quantities $u_{12}, v_{12}$, and $w_{12}$ of $\mathrm{BH}$ and the components $r_{1}, r_{2}$, and $r_{3}$ of GLL can now be made. The correspondence between the notation of GLL (on the left) and that of $\mathrm{BH}$ is

$$
\begin{aligned}
& \mathcal{E}_{1}=E_{2}, \\
& \mathcal{E}_{2}=E_{1}, \\
& p_{n 2}=\mu_{31}, \\
& p_{1 n}=\mu_{23}, \\
& \omega_{2}-\Omega_{n 2}=\Delta .
\end{aligned}
$$

Using Eq. (10d) with (9) and (11), we can express the GLL precession frequency $\Gamma$ in the $\mathrm{BH}$ notation as

$$
\Gamma=\left(\alpha^{2}+\beta^{2}\right) /|\Delta| \text {. }
$$

Since GLL assume that

$$
\Gamma \ll|\Delta|
$$

we may expand Eq. (4d) of $\mathrm{BH}$

$$
\delta=|\Delta| / 2+\left(\alpha^{2}+\beta^{2}\right) /|\Delta|+\cdots,
$$

which implies that the frequency $\delta+\Delta / 2$ of $\mathrm{BH}$ is given to a good approximation in the adiabatic limit by

$$
\delta+\Delta / 2 \approx \Gamma
$$

and that

$$
\delta \approx|\Delta| / 2 \text {. }
$$

Furthermore, the $\sin \theta$ and $\cos \theta$ of GLL can be expressed in the $\mathrm{BH}$ notation as

$$
\begin{aligned}
& \sin \theta=2 \alpha \beta /\left(\alpha^{2}+\beta^{2}\right) . \\
& \cos \theta=\left(\alpha^{2}-\beta^{2}\right) /\left(\alpha^{2}+\beta^{2}\right) .
\end{aligned}
$$

Using Eqs. (13) and (14), we can rewrite Eq. (6) to a very good approximation as

$$
\begin{aligned}
& u_{12}(t)=-\sin \theta \cos \theta(\cos \Gamma t-1), \\
& v_{12}(t)=-\sin \theta \sin \Gamma t, \\
& w_{12}(t)=1+\sin ^{2} \theta(\cos \Gamma t-1),
\end{aligned}
$$

where we have assumed at $t=0$ all of the atoms were in the ground state, i.e., $w_{12}(0)=1$. Thus, we can now join together the two approaches by the simple relationship

$$
\begin{aligned}
& u_{12}(t)=r_{1}(t), \\
& v_{12}(t)=r_{2}(t), \\
& w_{12}(t)=-r_{3}(t) .
\end{aligned}
$$

The difference in sign in (16c) is due to the fact that levels 1 and 2 of Fig. $1(\mathrm{BH})$ are reversed in Fig. 3 (GLL).

Thus, we have achieved our goal by making the simple connection of Eq. (16) between the BH and GLL results. This correspondence is consistent with the definitions of these quantities in terms of the density matrices defined above, Eqs. (4e)- $(4 \mathrm{~g})$ of $\mathrm{BH}$ and Eqs. (8a) $-(8 \mathrm{c})$ of GLL. It follows in the limit of very little mixing of the intermediate state, the adiabatic states (the primed states) of GLL, are almost equivalent to the original basis states used by BH. Then, the double-primed density matrix of GLL is approximately the same as the slowly varying density matrix used by $\mathrm{BH}$.

From the preceding discussion the complementary relationship between the two papers is now clear. The exact pulse solution of $\mathrm{BH}$ applies to the case of a three-level atom subject to two counter-propagating pulses such that the twophoton resonance condition $\omega_{21}=\Omega_{1}+\Omega_{2}$ is satisfied. The pulses, which are of constant amplitude, may be applied adiabatically (large $\Delta$ ) or nonadiabatically (small $\Delta$ ) with respect to the frequency offset $\Delta$ of the intermediate state. Frequency switching the laser or Stark switching the resonance line will satisfy the constant amplitude requirement of the $\mathrm{BH}$ solution. An excellent experimental demonstration of the utility of this technique and of the validity of the above solution has been the observation of Raman beats by Shoemaker and Brewer. ${ }^{3}$ On the other hand, the twophoton vector model of GLL can handle an arbitrary number of intermediate states and does not require the two-photon resonance condition to be satisfied, i.e., the two-photon frequency offset $\delta=\Omega_{12}-\omega_{1}-\omega_{2}$ does not have to equal zero. Furthermore, the GLL model can handle shapes that 
vary in time and thus is well adapted to the study of propagation problems and pulse reshaping effects. However, the GLL model is an approximation which requires that near-resonant pulses be applied adiabatically with respect to the intermediate states. The recent observation of twophoton optical nutation and free-induction decay by Loy $^{4}$ has verified in quantitative detail the GLL model. In summary, in their region of overlap, the results of $\mathrm{BH}$ and GLL are equivalent, but each approach can handle situations not describable by the other.

\section{ACKNOWLEDGMENTS}

The authors acknowledge careful reading of the manuscript by J. A. Armstrong, M. M. T. Loy, A. Z. Genack, and J. J. Wynne. Many useful discussions were held by one of us (D.G.) with M. M. T. Loy.
*Work partially supported by the U.S. Office of Naval Research.

†Work sponsored in part by the U.S. Office of Naval Research under Contract No. N00014-72-C-0153.

${ }^{1}$ R. G. Brewer and E. L. Hahn, Phys. Rev. A 11,1641 (1975).
${ }^{2}$ D. Grischkowsky, M. M. T. Loy, and P. F. Liao, Phys. Rev. A 12, 2514 (1975).

${ }^{3}$ R. L. Shoemaker and R. G. Brewer, Phys. Rev. Lett. 28,1430 (1972).

${ }^{4}$ M. M. T. Loy, Phys. Rev. Lett. 36, 1454 (1976). 